moderate to marked hyperemia (Power Doppler grade 2 and 3). Levels of sVCAM-1 $(r=0.20, p=0.028)$, Tie-2 $(r=0.28, p=0.001)$, and Angiostatin ( $r=0.25$, $\mathrm{p}=0.006$ ) correlated with a global arthritis sum score, defined by the sum of the semiquantitative PDUS scores for all joints examined.

Among the 81 patients with a DAS28-CRP $\leq 3.2,22$ patients had synovial hyperemia detected on at least one joint (Power Doppler grade 1 in 13 patients, grade 2 in 6 patients and grade 3 in 3 patients). Patients with synovial hyperemia on at least one joint were more likely to have significantly increased levels of PIGF (18.9 $\pm 11.2 \mathrm{pg} / \mathrm{mL}$ vs. $13.1 \pm 9.5 \mathrm{pg} / \mathrm{mL}, \mathrm{p}=0.022)$ and Tie-2 $(15.7 \pm 5.8 \mathrm{ng} / \mathrm{mL}$ vs. 12.6 $\pm 3.4 \mathrm{ng} / \mathrm{mL}, p=0.004$ ) than patients with absence of synovial hyperemia.

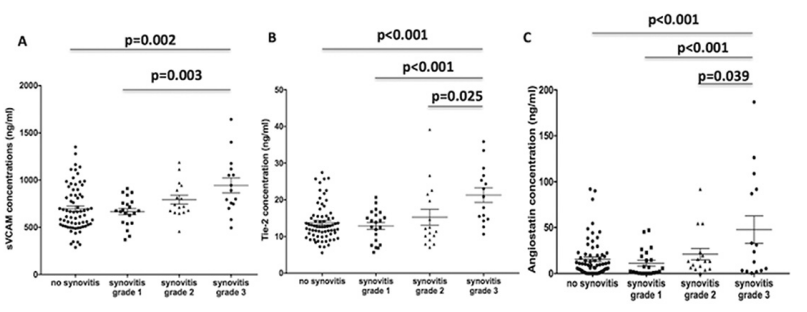

Conclusions: Serum levels of the angiogenic markers Tie-2, sVCAM-1 and Angiostatin were strongly associated with synovial vascularisation and inflammation assessed by PDUS among patients with established RA. Moreover, Tie-2 and PIGF were associated with persistent disease activity in RA patients in mow disease activity. These findings suggest that it may possible to find surrogate serum angiogenic biomarkers of active synovitis that might replace PDUS examination, in case of further confirmation of their pertinence.

Disclosure of Interest: A. Leblond: None declared, S. Pezet: None declared, A. P. Trouvin: None declared, M. Elhai: None declared, V. Gonzalez: None declared, Y. Allanore Grant/research support from: Pfizer, J. Avouac: None declared DOI: 10.1136/annrheumdis-2018-eular.2709

\section{THU0114 INFLUENCE OF AUTOANTIBODY STATUS IN TIME TO INITIATE DMARDS IN PATIENTS WITH EARLY RHEUMATOID ARTRHITIS}

J. Molina Collada, M.G. González Álvarez, V. Navarro-Compán, L. Nuño Nuño, D. Peiteado, A. Villalba, P. Bogas, A. Balsa. Rheumatology, Hospital La Paz, Madrid, Spain

Background: Factors contributing to an early referral to Rheumatologist and DMARDs initiation following symptom onset in rheumatoid arthritis (RA) patients are unclear. Recent data suggest that ACPA/RF double seropositivity is associated with delayed presentation to primary care and DMARDs initiation. Identification of these factors is mandatory to faciliate an early diagnosis and treatment of RA patients.

Objectives: To determine whether time to DMARDs initiation and time to first visit at early arthritis clinic (EAC) following symptoms onset differs between RA patients according to autoantibody status.

Methods: A prospective analysis of an EAC cohort including 1377 referred patients from 1993 to 2017 was undertaken for this study. Patients diagnosed of RA (according to physician's diagnosis) were selected. Based on the serological status, we clasified patients in 4 groups: $R F+/ A C P A+, R F-/ A C P A+, R F+/ A C P A-$, RF-/ACPA-. A baseline clinical assessment was completed including time (months) from symptoms onset to first assessment at EAC and to DMARD initiation. First, differences between serotypes were tested using chi-squared and student-t tests. Second, univariable and multivariable logistic regression models taking into account confounding factors (age, smoking and baseline DAS28)were employed to evaluate the association between autoantibody status and both periods: time to first visit at EAC and to DMARDs initiation.

Results: A total of 463 RA patients were included for analysis, of whom 292 (63.1\%) were RF/ACPA double-seropositive (RF+/ACPA+),35 (7.6\%)RF-/ACPA +,39 (8.4\%) RF+/ACPA- and 92 (19.9\%)RF-/ACPA-. Demographic and clinical data are shown in table 1 . In the univariate analysis, statiscally significant differences were observed for both periods when patients were stratified by autoantibody serotype, where $\mathrm{RF}+\mathrm{ACPA}+$ individuals experienced the longer delay to presentation at EAC compared with RF-/ACPA- $(5.0 \pm 4.6$ vs $3.5 \pm 3.4$ months; $p<0.05)$. RF +/ACPA +patients experienced also significantly longer symptom duration before DMARD initiation than RF-/ACPA- $(7.4 \pm 11.2$ vs $4.9 \pm 5$ months; $p<0.05)$. In the univariable analysis, autoabtibody status (doble seropositive and RF-/ACPA +vsRF-/ ACPA-) was signficantly asssociated with time to DMARDs initiation. However, after adjusting by confounding factors in the multivariable analysis, the autoantibody status did not remain significantly associated with time to initiate DMARDs (RF+/ACPA-; $30.186 ; p=0.1, R F-/ A C P A+; B 0.112 ; p=0.3, R F+/ A C P A+; B 0.228$; $\mathrm{p}=0.1$ )

\begin{tabular}{lccc}
\hline & Beta & \multicolumn{2}{c}{$95 \%$ C.I. } \\
\cline { 3 - 4 } & & Lower & Upper \\
\hline Non adjusted &, 035 & $-2,462$ & 4944 \\
RFpositive &, $\mathbf{1 0 4}$ &, 038 & 7736 \\
ACPApositive &, 120 &, $\mathbf{1 5 7}$ & 4791 \\
$\begin{array}{l}\text { Double- } \\
\text { seropositive }\end{array}$ & & & \\
Adjusted & & $-4,952$ & 26414 \\
RF positive &, 186 & $-14,512$ & 37175 \\
ACPA positive &, 112 & $-2,520$ & 20308 \\
Double- &, 228 & $-7,575$ &, 144 \\
seropositive &,- 239 &,- 384 &, 207 \\
DAS 28 &,- 073 & $-14,996$ & 9118 \\
Age &,- 066 & $-19,051$ & 10942 \\
Smoker &,- 081 & & \\
Former smoker & & & \\
\hline
\end{tabular}

Abstract THU0114 - Table 1. Baseline characteristics of patients, stratified by autoantibody status

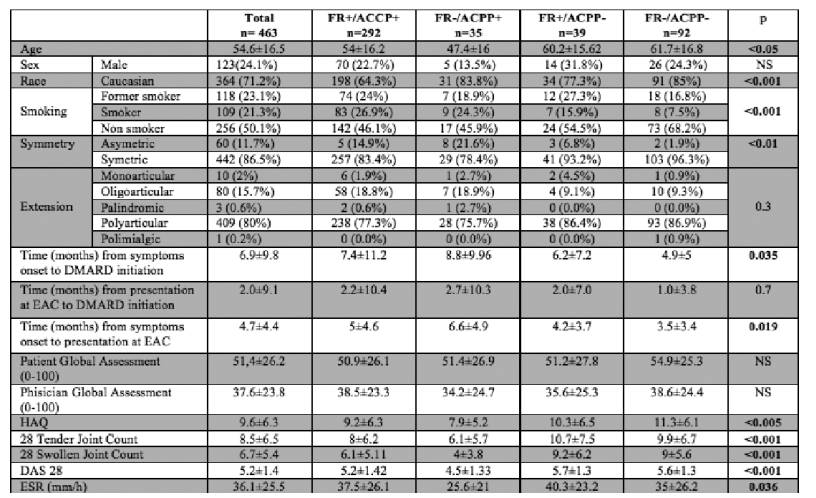

Conclusions: In our EAC cohort, time to EAC presentation and DMARD initiation following symptom onset in early RA does not differ according to patients' autoantibody status. However, more studies with greater sample size are necessary to confirm these results.

Disclosure of Interest: None declared

DOI: 10.1136/annrheumdis-2018-eular.5269

\section{THU0115 PSYCHOLOGICAL AND FUNCTIONAL STATES PREDICT DISEASE FLARE FOLLOWING TNF INHIBITOR TAPERING IN PATIENTS WITH RHEUMATOID ARTHRITIS: A POST-HOC ANALYSIS OF DATA FROM THE OPTIMISINGTNF TAPERING IN RA (OPTTIRA) COHORT}

K. Bechman, F.E. Sin, F. Ibrahim, S. Norton, D. Scott, A. Cope, J. Galloway. Rheumatology, King's College London, London, UK

Background: Tapering or discontinuation of anti-TNF therapy appears to be feasible, safe and effective in a selected proportion of Rheumatoid Arthritis (RA) patients. Depression is highly prevalent in RA and may impact on flare incidence through a number of mechanisms. It is an independent predictor for flare in patients with active disease and is negatively associated with remission. ${ }^{1}$ To date, there are no studies directly addressing the role of depression, anxiety or low mood in predicting flares in patients tapering their biological therapy.

Objectives: To investigate if psychological and functional states predict flare in RA patients with low disease activity (LDA) or in remission who undergo treatment tapering of their anti-TNF agents.

Methods: This study is a post-hoc analysis of the OPTTIRA trial, ${ }^{2}$ a multi-centre, prospective, randomised, open label study investigating anti-TNF tapering in established RA patients in sustained LDA. Baseline patient-reported outcomes including HAQ-DI, EQ-5D, FACIT-F, and SF-36 including the Mental Health Index (MHI) component were collected. The $\mathrm{MHI}$ has been validated as a screening tool for depression in RA patients. ${ }^{3}$ The primary outcome was flare, defined as an increase in DAS28 $\geq 0.6$, and at least one additional swollen joint. Logistic regression was used to identify patient-reported outcomes that predict flare, adjusting for baseline covariates (age, gender, treatment arm, DAS28 and BMI).

Results: 97 were randomised into a tapering arm, either by $33 \%$ or $66 \%$ of their anti-TNF dose. The majority of patients were on methotrexate in combination with their anti-TNF therapy $(n=67,69 \%)$ and the median disease duration was 11 years [IQR: 7-17]. Seventy three $(75 \%)$ fulfilled DAS28 remission criteria (DAS28 <2.6). The median SF36 MHI score was 84 . A score of $\leq 56$, the cut off used to detect depression was observed in $11 \%$ of patients. Forty one patients $(42 \%)$ flared. Baseline DAS28 score was associated with flare, remaining significant after 